\title{
Oscillation Criteria for Nonlinear Third-Order Neutral Dynamic Equations with Damping on Time Scales
}

\author{
Yang-Cong Qiu, ${ }^{1}$ Akbar Zada, ${ }^{2}$ Haiyong Qin, ${ }^{3}$ and Tongxing $\mathrm{Li}^{4}$ \\ ${ }^{1}$ School of Humanities and Social Science, Shunde Polytechnic, Foshan, Guangdong 528333, China \\ ${ }^{2}$ Department of Mathematics, University of Peshawar, Peshawar 25000, Pakistan \\ ${ }^{3}$ School of Mathematics, Qilu Normal University, Jinan, Shandong 250013, China \\ ${ }^{4}$ School of Information Science and Engineering, Linyi University, Linyi, Shandong 276005, China
}

Correspondence should be addressed to Tongxing Li; litongx2007@163.com

Received 12 May 2017; Accepted 26 July 2017; Published 28 August 2017

Academic Editor: Xinguang Zhang

Copyright (c) 2017 Yang-Cong Qiu et al. This is an open access article distributed under the Creative Commons Attribution License, which permits unrestricted use, distribution, and reproduction in any medium, provided the original work is properly cited.

We establish several oscillation criteria for a class of third-order nonlinear dynamic equations with a damping term and a nonpositive neutral coefficient by using the Riccati transformation. Two illustrative examples are presented to show the significance of the results obtained.

\section{Introduction}

In this paper, we are concerned with the oscillation of a class of third-order damped dynamic equations of neutral type

$$
\begin{gathered}
\left(r(t) \varphi_{\gamma}\left(z^{\Delta \Delta}(t)\right)\right)^{\Delta}+d(t) \varphi_{\gamma}\left(z^{\Delta \Delta}(t)\right) \\
+f(t, x(h(t)))=0
\end{gathered}
$$

on a time scale $\mathbb{T}$ satisfying sup $\mathbb{T}=\infty$, where $t \in\left[t_{0}, \infty\right)_{\mathbb{T}}$, $\varphi_{\gamma}(u)=|u|^{\gamma-1} u$, and $z(t)=x(t)-p(t) x(g(t))$. Throughout, we suppose that the following conditions are satisfied:

$\left(C_{1}\right) \gamma \geq 1$ is a constant.

$\left(C_{2}\right) r \in C_{r d}\left(\left[t_{0}, \infty\right)_{\mathbb{T}},(0, \infty)\right), d \in C_{r d}\left(\left[t_{0}, \infty\right)_{\mathbb{T}}, \mathbb{R}\right),-d / r$ is positively regressive (i.e., $r(t)-(\sigma(t)-t) d(t)>0$ ), and

$$
\int_{t_{0}}^{\infty}\left(\frac{e_{-d / r}\left(t, t_{0}\right)}{r(t)}\right)^{1 / \gamma} \Delta t=\infty .
$$

$\left(C_{3}\right) p \in C_{r d}\left(\left[t_{0}, \infty\right)_{\mathbb{T}},[0, \infty)\right)$ and $\lim _{t \rightarrow \infty} p(t)=p_{0}$, where $0 \leq p_{0}<1$.

$\left(C_{4}\right) g, h \in C_{r d}\left(\left[t_{0}, \infty\right)_{\mathbb{T}}, \mathbb{T}\right), g(t) \leq t, h(t) \leq t$ or $h(t) \geq t$, $\lim _{t \rightarrow \infty} g(t)=\lim _{t \rightarrow \infty} h(t)=\infty$, and there exists a sequence $\left\{c_{k}\right\}_{k \geq 0}$ such that $\lim _{k \rightarrow \infty} c_{k}=\infty$ and $g\left(c_{k+1}\right)=c_{k}$.

$\left(C_{5}\right) f \in C\left(\left[t_{0}, \infty\right)_{\mathbb{T}} \times \mathbb{R}, \mathbb{R}\right)$ and there exists a function $q \in C_{r d}\left(\left[t_{0}, \infty\right)_{\mathbb{T}},(0, \infty)\right)$ such that $u f(t, u)>0$ and $f(t, u) /\left(|u|^{\gamma-1} u\right) \geq q(t)$ for $u \neq 0$.

The theory of time scales, which was firstly introduced by Hilger in $[1,2]$, has been enriched by researchers; see, for instance, $[3,4]$, monographs $[5,6]$, and the references cited therein. During the past decade, a great deal of interest in oscillation of solutions to different classes of dynamic equations on time scales has been shown; we refer the reader to [7-23].

Yu and Wang [23] studied a third-order dynamic equation

$$
\begin{aligned}
& \left(\frac{1}{a_{2}(t)}\left(\left(\frac{1}{a_{1}(t)}\left(x^{\Delta}(t)\right)^{\alpha_{1}}\right)^{\Delta}\right)^{\alpha_{2}}\right)^{\Delta}+q(t) f(x(t)) \\
& \quad=0, \quad \alpha_{1} \alpha_{2}=1 .
\end{aligned}
$$

Agarwal et al. [8, 10], Candan [12], Erbe et al. [13], Hassan [15], and $\mathrm{Li}$ et al. [18] considered a third-order retarded dynamic equation

$$
\left(a(t)\left[\left(r(t) x^{\Delta}(t)\right)^{\Delta}\right]^{\gamma}\right)^{\Delta}+f(t, x(\tau(t)))=0 .
$$


Saker et al. [21] studied a second-order damped dynamic equation

$$
\left(a(t) x^{\Delta}(t)\right)^{\Delta}+p(t) x^{\Delta^{\sigma}}(t)+q(t)\left(f \circ x^{\sigma}\right)=0
$$

whereas Qiu and Wang [20] considered a second-order damped dynamic equation

$$
\begin{aligned}
& \left(r(t) \varphi_{\gamma}\left(x^{\Delta}(t)\right)\right)^{\Delta}+p(t) \varphi_{\gamma}\left(x^{\Delta}(t)\right)+f(t, x(g(t))) \\
& \quad=0,
\end{aligned}
$$

where $\gamma>0, \varphi_{\gamma}(u)=|u|^{\gamma-1} u$, and

$$
g(t) \geq \begin{cases}\sigma(t), & 0<\gamma<1 \\ t, & \gamma \geq 1\end{cases}
$$

Han et al. [14] and Qiu [19] investigated the third-order dynamic equations with nonpositive neutral coefficients

$$
\begin{array}{r}
\left(r(t) z^{\Delta \Delta}(t)\right)^{\Delta}+q(t) x^{\gamma}(h(t))=0, \\
r^{\Delta} \geq 0, \\
\left(r_{1}(t)\left(\left(r_{2}(t)\left(z^{\Delta}(t)\right)^{\gamma_{2}}\right)^{\Delta}\right)^{\gamma_{1}}\right)^{\Delta}+f(t, x(h(t)))=0,
\end{array}
$$

respectively, where $z(t)=x(t)-p(t) x(g(t))$.

In this paper, using the Riccati transformation, we obtain some sufficient conditions which ensure that every solution $x$ of (1) either is oscillatory or converges to a finite number asymptotically. We do not impose restrictive assumption $d \geq 0$ in our results. To illustrate the significance of new results, two examples are provided in the last section. In what follows, all functional inequalities are assumed to hold for all sufficiently large $t$. Without loss of generality, we can deal only with eventually positive solutions of (1).

Definition 1. A solution $x$ of (1) is said to be oscillatory if it is neither eventually positive nor eventually negative; otherwise, it is termed nonoscillatory.

Definition 2. Equation (1) is said to be almost oscillatory if all its solutions either are oscillatory or converge to zero asymptotically.

\section{Auxiliary Results}

Lemma 3 (see [19, Lemma 2.1]). Suppose that $x$ is an eventually positive solution of (1) and there exists a constant $a \geq 0$ such that $\lim _{t \rightarrow \infty} z(t)=a$. Then

$$
\lim _{t \rightarrow \infty} x(t)=\frac{a}{1-p_{0}}
$$

Lemma 4. If $x$ is an eventually positive solution of (1), then there exists a sufficiently large $T \in\left[t_{0}, \infty\right)_{\mathbb{T}}$ such that, for $t \in$ $[T, \infty)_{\mathbb{T}}$,

$$
\begin{gathered}
{\left[\frac{r(t)\left(z^{\Delta \Delta}(t)\right)^{\gamma}}{e_{-d / r}(t, T)}\right]^{\Delta}<0, \quad z^{\Delta \Delta}(t)>0} \\
z^{\Delta}(t)>0 \\
\text { or } z^{\Delta}(t)<0 .
\end{gathered}
$$

Proof. Let $x$ be an eventually positive solution of (1). From $\left(C_{3}\right)$ and $\left(C_{4}\right)$, there exist a $t_{1} \in\left[t_{0}, \infty\right)_{\mathbb{T}}$ and a constant $p_{1}$ such that $p_{0}<p_{1}<1, x(t)>0, x(g(t))>0, x(h(t))>0$, and $p(t) \leq p_{1}$ for $t \in\left[t_{1}, \infty\right)_{\mathbb{T}}$. By virtue of $(1)$ and $\left(C_{5}\right)$, we conclude that

$$
\left[\frac{r(t) \varphi_{\gamma}\left(z^{\Delta \Delta}(t)\right)}{e_{-d / r}\left(t, t_{1}\right)}\right]^{\Delta}=-\frac{f(t, x(h(t)))}{e_{-d / r}\left(\sigma(t), t_{1}\right)}<0 .
$$

We claim that there exists a $t_{2} \in\left[t_{1}, \infty\right)_{\mathbb{T}}$ such that $z^{\Delta \Delta}(t)>$ 0 for $t \in\left[t_{2}, \infty\right)_{\mathbb{T}}$. Otherwise, assume that $z^{\Delta \Delta}(t)<0$ for $t \in\left[t_{2}, \infty\right)_{\mathbb{T}}$. Then, by (12) and $\left(C_{2}\right), \lim _{t \rightarrow \infty} z^{\Delta}(t)=-\infty$. It follows from $z^{\Delta \Delta}(t)<0$ and $z^{\Delta}(t)<0$ that $\lim _{t \rightarrow \infty} z(t)=$ $-\infty$. Hence, there exists a $t_{3} \in\left[t_{2}, \infty\right)_{\mathbb{W}}$ such that $z(t)<0$ for $t \in\left[t_{3}, \infty\right)_{\mathbb{T}}$; that is,

$$
x(t)<p(t) x(g(t)) \leq p_{1} x(g(t)), \quad t \in\left[t_{3}, \infty\right)_{\mathbb{T}} .
$$

By $\left(C_{4}\right)$, there exists a sufficiently large integer $k_{0}$ such that $c_{k} \in\left[t_{3}, \infty\right)_{\mathbb{T}}$ for $k \geq k_{0}$. For $k \geq k_{0}+1$, we have

$$
\begin{aligned}
x\left(c_{k}\right) & <p_{1} x\left(g\left(c_{k}\right)\right)=p_{1} x\left(c_{k-1}\right)<p_{1}^{2} x\left(g\left(c_{k-1}\right)\right) \\
& =p_{1}^{2} x\left(c_{k-2}\right)<\cdots<p_{1}^{k-k_{0}} x\left(g\left(c_{k_{0}+1}\right)\right) \\
& =p_{1}^{k-k_{0}} x\left(c_{k_{0}}\right),
\end{aligned}
$$

which yields $\lim _{k \rightarrow \infty} x\left(c_{k}\right)=0$ and so $\lim _{k \rightarrow \infty} z\left(c_{k}\right)=0$, which contradicts the fact that $\lim _{t \rightarrow \infty} z(t)=-\infty$. Therefore, $z^{\Delta \Delta}(t)>0$ and hence $z^{\Delta}(t)>0$ or $z^{\Delta}(t)<0$. The proof is complete.

Lemma 5 (see [19, Lemma 2.3]). If $x$ is an eventually positive solution of (1), then $z$ is eventually positive or $\lim _{t \rightarrow \infty} x(t)=0$.

Lemma 6. Let $x$ be an eventually positive solution of (1) and suppose that $z$ and $z^{\Delta}$ are eventually positive. Assume also that

$$
\int_{t_{0}}^{\infty} \frac{q(t) h^{\gamma}(t)}{e_{-d / r}\left(\sigma(t), t_{0}\right)} \Delta t=\infty
$$

Then there exists a sufficiently large $T \in\left[t_{0}, \infty\right)_{\mathbb{W}}$ such that, for $t \in[T, \infty)_{\mathbb{T}}$,

$$
\frac{z(h(t))}{z(t)} \geq \min \left\{\frac{h(t)}{t}, 1\right\} .
$$


Proof. Let $x$ be an eventually positive solution of (1) and assume that there exists a $t_{1} \in\left[t_{0}, \infty\right)_{\mathbb{T}}$ such that $z(t)>0$ and $z^{\Delta}(t)>0$ for $t \in\left[t_{1}, \infty\right)_{\mathbb{T}}$. Define

$$
y(t)=z(t)-t z^{\Delta}(t), \quad t \in\left[t_{1}, \infty\right)_{\mathbb{V}} .
$$

Then, by Lemma 4 , for $t \in\left[t_{1}, \infty\right)_{\mathbb{T}}$,

$$
\begin{aligned}
y^{\Delta}(t) & =z^{\Delta}(t)-\left(z^{\Delta}(t)+\sigma(t) z^{\Delta \Delta}(t)\right) \\
& =-\sigma(t) z^{\Delta \Delta}(t)<0 .
\end{aligned}
$$

We can prove that $y$ is eventually positive. If not, then there exists a $t_{2} \in\left[t_{1}, \infty\right)_{\mathbb{T}}$ such that $y(t)<0$ for $t \in\left[t_{2}, \infty\right)_{\mathbb{T}}$. Hence, we conclude that

$$
\begin{aligned}
&\left(\frac{z(t)}{t}\right)^{\Delta}=\frac{t z^{\Delta}(t)-z(t)}{t \sigma(t)}=-\frac{y(t)}{t \sigma(t)}>0, \\
& t \in\left[t_{2}, \infty\right)_{\mathbb{T}},
\end{aligned}
$$

which implies that $z(t) / t$ is strictly increasing on $\left[t_{2}, \infty\right)_{\mathbb{T}}$. Since $\lim _{t \rightarrow \infty} h(t)=\infty$, there exists a $t_{3} \in\left[t_{2}, \infty\right)_{\mathbb{T}}$ such that, for $t \in\left[t_{3}, \infty\right)_{\mathbb{T}}, h(t) \geq h\left(t_{3}\right) \geq t_{2}$, and so

$$
\frac{z(h(t))}{h(t)} \geq \frac{z\left(h\left(t_{3}\right)\right)}{h\left(t_{3}\right)} .
$$

Using $\left(C_{5}\right)$, we have $f(t, x(h(t))) \geq q(t) x^{\gamma}(h(t)) \geq$ $q(t) z^{\gamma}(h(t))$. Therefore, by virtue of (1) and Lemma 4 , for $t \in\left[t_{3}, \infty\right)_{\mathbb{T}}$,

$$
\begin{aligned}
{\left[\frac{r(t)\left(z^{\Delta \Delta}(t)\right)^{\gamma}}{e_{-d / r}\left(t, t_{3}\right)}\right]^{\Delta} } & =-\frac{f(t, x(h(t)))}{e_{-d / r}\left(\sigma(t), t_{3}\right)} \\
& \leq-\frac{q(t) z^{\gamma}(h(t))}{e_{-d / r}\left(\sigma(t), t_{3}\right)}
\end{aligned}
$$

Integrating (21) from $t_{3}$ to $t$, we get

$$
\begin{aligned}
& \frac{r(t)\left(z^{\Delta \Delta}(t)\right)^{\gamma}}{e_{-d / r}\left(t, t_{3}\right)}-r\left(t_{3}\right)\left(z^{\Delta \Delta}\left(t_{3}\right)\right)^{\gamma} \\
& \leq-\int_{t_{3}}^{t} \frac{q(s) z^{\gamma}(h(s))}{e_{-d / r}\left(\sigma(s), t_{3}\right)} \Delta s \\
& \leq-\frac{z^{\gamma}\left(h\left(t_{3}\right)\right)}{h^{\gamma}\left(t_{3}\right)} \int_{t_{3}}^{t} \frac{q(s) h^{\gamma}(s)}{e_{-d / r}\left(\sigma(s), t_{3}\right)} \Delta s .
\end{aligned}
$$

It follows from (15) that

$$
\begin{aligned}
& r\left(t_{3}\right)\left(z^{\Delta \Delta}\left(t_{3}\right)\right)^{\gamma} \geq \frac{z^{\gamma}\left(h\left(t_{3}\right)\right)}{h^{\gamma}\left(t_{3}\right)} \int_{t_{3}}^{t} \frac{q(s) h^{\gamma}(s)}{e_{-d / r}\left(\sigma(s), t_{3}\right)} \Delta s \longrightarrow \\
& \infty, \quad t \longrightarrow \infty,
\end{aligned}
$$

which is a contradiction. Hence, $y$ is eventually positive. Then, there exists a sufficiently large $T \in\left[t_{1}, \infty\right)_{\mathbb{T}}$ such that, for $t \in[T, \infty)_{\mathbb{T}}$,

$$
\left(\frac{z(t)}{t}\right)^{\Delta}=-\frac{y(t)}{t \sigma(t)}<0,
$$

so $z(t) / t$ is strictly decreasing on $[T, \infty)_{\mathbb{T}}$. If $h(t) \geq t$, then $z(h(t)) / z(t) \geq 1$. If $h(t) \leq t$, then $z(h(t)) / z(t) \geq h(t) / t$. Therefore, we arrive at (16). This completes the proof.

Lemma 7. Assume that all assumptions of Lemma 6 are satisfied. For $t \in\left[t_{1}, \infty\right)_{\mathbb{T}}$, define

$$
u(t)=A(t) \frac{r(t)\left(z^{\Delta \Delta}(t)\right)^{\gamma}}{e_{-d / r}\left(t, t_{1}\right) z^{\gamma}(t)},
$$

where $A \in C_{r d}^{1}\left(\left[t_{1}, \infty\right)_{\mathbb{T}},(0, \infty)\right)$. Then, u satisfies

$$
u^{\Delta}(t)+\frac{A(t) \alpha(t) q(t)}{e_{-d / r}\left(\sigma(t), t_{1}\right)}-\Phi(t) \leq 0,
$$

where

$$
\begin{aligned}
\Phi(t)= & A^{\Delta}(t)\left(\frac{u}{A}\right)^{\sigma}(t) \\
& -\gamma A(t) \delta(t)\left[\left(\frac{u}{A}\right)^{\sigma}\right]^{(1+\gamma) / \gamma}(t), \\
\alpha(t)= & \min \left\{\left(\frac{h(t)}{t}\right)^{\gamma}, 1\right\}, \\
\delta(t)= & \int_{t_{1}}^{t}\left(\frac{e_{-d / r}\left(s, t_{1}\right)}{r(s)}\right)^{1 / \gamma} \Delta s .
\end{aligned}
$$

Proof. Suppose that all assumptions of Lemma 6 hold. Differentiating (25) and using (1), we have

$$
\begin{gathered}
u^{\Delta}(t)=\frac{A(t)}{z^{\gamma}(t)}\left(\frac{r\left(z^{\Delta \Delta}\right)^{\gamma}}{e_{-d / r}\left(\cdot, t_{1}\right)}\right)^{\Delta}(t)+\left(\frac{A}{z^{\gamma}}\right)^{\Delta}(t) \\
\cdot\left(\frac{r\left(z^{\Delta \Delta}\right)^{\gamma}}{e_{-d / r}\left(\cdot, t_{1}\right)}\right)^{\sigma}(t)=-\frac{A(t)}{z^{\gamma}(t)} \frac{f(t, x(h(t)))}{e_{-d / r}\left(\sigma(t), t_{1}\right)} \\
+\frac{A^{\Delta}(t) z^{\gamma}(t)-A(t)\left(z^{\gamma}\right)^{\Delta}(t)}{z^{\gamma}(t) z^{\gamma}(\sigma(t))}\left(\frac{r\left(z^{\Delta \Delta}\right)^{\gamma}}{e_{-d / r}\left(\cdot, t_{1}\right)}\right)^{\sigma} \\
\cdot(t) .
\end{gathered}
$$

If $h(t) \geq t$, then

$$
\begin{aligned}
f(t, x(h(t))) & \geq q(t) x^{\gamma}(h(t)) \geq q(t) z^{\gamma}(h(t)) \\
& \geq q(t) z^{\gamma}(t) .
\end{aligned}
$$

Assume now that $h(t) \leq t$. It follows from Lemma 6 that

$$
\begin{aligned}
f(t, x(h(t))) & \geq q(t) z^{\gamma}(h(t)) \\
& \geq\left(\frac{h(t)}{t}\right)^{\gamma} q(t) z^{\gamma}(t) .
\end{aligned}
$$

Hence, we have

$$
\begin{aligned}
u^{\Delta}(t) \leq & -\frac{A(t) \alpha(t) q(t)}{e_{-d / r}\left(\sigma(t), t_{1}\right)}+A^{\Delta}(t)\left(\frac{u}{A}\right)^{\sigma}(t) \\
& -A(t) \frac{\left(z^{\gamma}\right)^{\Delta}(t)}{z^{\gamma}(t)}\left(\frac{u}{A}\right)^{\sigma}(t) .
\end{aligned}
$$


Using Pötzsche chain rule (see [5, Theorem 1.90] for details), we obtain

$$
\left(z^{\gamma}\right)^{\Delta}(t) \geq \gamma z^{\gamma-1}(t) z^{\Delta}(t)
$$

which yields

$$
\frac{\left(z^{\gamma}\right)^{\Delta}(t)}{z^{\gamma}(t)} \geq \frac{\gamma z^{\gamma-1}(t) z^{\Delta}(t)}{z^{\gamma}(t)}=\frac{\gamma z^{\Delta}(t)}{z(t)} .
$$

From Lemma 4, we conclude that

$$
\begin{aligned}
z^{\Delta}(t) & =z^{\Delta}\left(t_{1}\right)+\int_{t_{1}}^{t} z^{\Delta \Delta}(s) \Delta s \\
= & z^{\Delta}\left(t_{1}\right) \\
& +\int_{t_{1}}^{t}\left(\frac{r(s)\left(z^{\Delta \Delta}(s)\right)^{\gamma}}{e_{-d / r}\left(s, t_{1}\right)}\right)^{1 / \gamma}\left(\frac{e_{-d / r}\left(s, t_{1}\right)}{r(s)}\right)^{1 / \gamma} \Delta s \\
\geq & \left(\frac{r(t)\left(z^{\Delta \Delta}(t)\right)^{\gamma}}{e_{-d / r}\left(t, t_{1}\right)}\right)^{1 / \gamma} \int_{t_{1}}^{t}\left(\frac{e_{-d / r}\left(s, t_{1}\right)}{r(s)}\right)^{1 / \gamma} \Delta s \\
= & \delta(t)\left(\frac{r(t)\left(z^{\Delta \Delta}(t)\right)^{\gamma}}{e_{-d / r}\left(t, t_{1}\right)}\right)^{1 / \gamma},
\end{aligned}
$$

which implies that

$$
\begin{aligned}
\frac{z^{\Delta}(t)}{z(t)} & \geq \delta(t)\left(\frac{r(t)\left(z^{\Delta \Delta}(t)\right)^{\gamma}}{e_{-d / r}\left(t, t_{1}\right)}\right)^{1 / \gamma} \frac{1}{z(t)} \\
& \geq \delta(t)\left[\left(\frac{r\left(z^{\Delta \Delta}\right)^{\gamma}}{e_{-d / r}\left(\cdot, t_{1}\right)}\right)^{1 / \gamma}\right]^{\sigma}(t) \frac{1}{z^{\sigma}(t)} \\
& =\delta(t)\left[\left(\frac{u}{A}\right)^{\sigma}\right]^{1 / \gamma}(t) .
\end{aligned}
$$

It follows now from (31) that

$$
\begin{aligned}
u^{\Delta}(t) \leq & -\frac{A(t) \alpha(t) q(t)}{e_{-d / r}\left(\sigma(t), t_{1}\right)}+A^{\Delta}(t)\left(\frac{u}{A}\right)^{\sigma}(t) \\
& -\gamma A(t) \delta(t)\left[\left(\frac{u}{A}\right)^{\sigma}\right]^{(1+\gamma) / \gamma}(t) .
\end{aligned}
$$

The proof is complete.

Lemma 8. Assume that $x$ is an eventually positive solution of (1) and $z^{\Delta}$ is eventually negative. If

$$
\int_{t_{0}}^{\infty} \frac{q(t)}{e_{-d / r}\left(\sigma(t), t_{0}\right)} \Delta t=\infty,
$$

then $\lim _{t \rightarrow \infty} x(t)=0$.

Proof. Since $z^{\Delta}$ is eventually negative, $z$ is either eventually positive or eventually negative. If $z$ is eventually negative, then there exist a constant $c<0$ and a $T \in\left[t_{0}, \infty\right)_{\mathbb{T}}$ such that $z(t)<c$ for $t \in[T, \infty)_{\mathbb{T}}$, which causes a contradiction as in the proof of Lemma 4 . Thus, $z$ is eventually positive.

Taking into account the fact that $x>0$, by Lemma 4 , there exists a $t_{1} \in\left[t_{0}, \infty\right)_{\mathbb{T}}$ such that $z^{\Delta \Delta}(t)>0$ for $t \in\left[t_{1}, \infty\right)_{\mathbb{T}}$. We prove that $\lim _{t \rightarrow \infty} z(t)=b \geq 0$. Otherwise, there exists a $t_{2} \in\left[t_{1}, \infty\right)_{\mathbb{T}}$ such that $z(t)<0$ for $t \in\left[t_{2}, \infty\right)_{\mathbb{T}}$, and a similar contradiction can be obtained. Suppose that $b>0$. It follows from the proof of Lemma 4 and $z(h(t))>b$ that

$$
\begin{aligned}
{\left[\frac{r(t)\left(z^{\Delta \Delta}(t)\right)^{\gamma}}{e_{-d / r}\left(t, t_{1}\right)}\right]^{\Delta} } & \leq-\frac{q(t) z^{\gamma}(h(t))}{e_{-d / r}\left(\sigma(t), t_{1}\right)} \\
& <-\frac{b^{\gamma} q(t)}{e_{-d / r}\left(\sigma(t), t_{1}\right)}
\end{aligned}
$$

Integrating (38) from $t_{1}$ to $t, t \in\left[\sigma\left(t_{1}\right), \infty\right)_{\mathbb{T}}$, we have

$$
\begin{aligned}
& \frac{r(t)\left(z^{\Delta \Delta}(t)\right)^{\gamma}}{e_{-d / r}\left(t, t_{1}\right)} \leq r\left(t_{1}\right)\left(z^{\Delta \Delta}\left(t_{1}\right)\right)^{\gamma} \\
& -b^{\gamma} \int_{t_{1}}^{t} \frac{q(s)}{e_{-d / r}\left(\sigma(s), t_{1}\right)} \Delta s \longrightarrow \\
& -\infty, \quad t \longrightarrow \infty,
\end{aligned}
$$

which contradicts the fact that $z^{\Delta \Delta}(t)>0$. Hence, $b=0$ and so $\lim _{t \rightarrow \infty} x(t)=0$ when using Lemma 3 . This completes the proof.

\section{Main Results}

Let $D=\left\{(t, s) \in \mathbb{T}^{2}: t \geq s \geq t_{0}\right\}$. Define

$$
\begin{aligned}
\mathscr{H} & =\left\{H(t, s) \in C_{r d}(D,[0, \infty)): H(t, t)\right. \\
& \left.=0, H(t, s)>0, H_{2}^{\Delta}(t, s) \leq 0, t>s \geq t_{0}\right\},
\end{aligned}
$$

where $H_{2}^{\Delta}$ is the $\Delta$-partial derivative of $H$ with respect to $s$.

Theorem 9. Assume that (15) holds and there exist two functions $A \in C_{r d}^{1}\left(\left[t_{0}, \infty\right)_{\mathbb{T}},(0, \infty)\right)$ and $H \in \mathscr{H}$ such that, for all sufficiently large $t_{1} \in\left[t_{0}, \infty\right)_{\mathbb{T}}$ and for some $t_{2} \in\left[t_{1}, \infty\right)_{\mathbb{T}}$,

$$
\begin{aligned}
& \limsup _{t \rightarrow \infty} \frac{1}{H\left(t, t_{2}\right)} \int_{t_{2}}^{t}\left[\frac{H(t, s) A(s) \alpha(s) q(s)}{e_{-d / r}\left(\sigma(s), t_{1}\right)}\right. \\
& \left.-\frac{1}{(1+\gamma)^{1+\gamma}}\right] \Delta s \\
& \left.\cdot \frac{\left(H_{2}^{\Delta}(t, s) A^{\sigma}(s)+H(t, s) A^{\Delta}(s)\right)^{1+\gamma}}{(H(t, s) A(s) \delta(s))^{\gamma}}\right] \Delta \\
& \quad=\infty,
\end{aligned}
$$

where $\alpha$ and $\delta$ are as in Lemma 7. Then every solution $x$ of (1) is oscillatory or $\lim _{t \rightarrow \infty} x(t)$ exists (finite). 
Proof. Suppose that (1) has a nonoscillatory solution $x$. Without loss of generality, let $x$ be eventually positive. From Lemma 5, it follows that $z$ is eventually positive or $\lim _{t \rightarrow \infty} x(t)=0$. Assume that $z$ is eventually positive. By Lemma 4 , there exists a $t_{1} \in\left[t_{0}, \infty\right)_{\mathbb{T}}$ such that either $z^{\Delta}(t)>$ 0 or $z^{\Delta}(t)<0$ for $t \in\left[t_{1}, \infty\right)_{\mathbb{T}}$. Let $z^{\Delta}(t)>0$ for $t \in\left[t_{1}, \infty\right)_{\mathbb{T}}$. Define $u$ by (25). Then, by Lemma 7, (26) holds. It follows from (26) that, for some $t_{2} \in\left[t_{1}, \infty\right)_{\mathbb{T}}$,

$$
\begin{aligned}
& \int_{t_{2}}^{t} \frac{H(t, s) A(s) \alpha(s) q(s)}{e_{-d / r}\left(\sigma(s), t_{1}\right)} \Delta s \leq-\int_{t_{2}}^{t} H(t, s) u^{\Delta}(s) \Delta s \\
& \quad+\int_{t_{2}}^{t} H(t, s) \Phi(s) \Delta s=H\left(t, t_{2}\right) u\left(t_{2}\right) \\
& \quad+\int_{t_{2}}^{t}\left(H_{2}^{\Delta}(t, s) u^{\sigma}(s)+H(t, s) \Phi(s)\right) \Delta s \\
& \quad=H\left(t, t_{2}\right) u\left(t_{2}\right)+\int_{t_{2}}^{t}\left(H_{2}^{\Delta}(t, s) A^{\sigma}(s)\left(\frac{u}{A}\right)^{\sigma}(s)\right. \\
& \quad+H(t, s) \Phi(s)) \Delta s,
\end{aligned}
$$

where

$$
\begin{aligned}
H_{2}^{\Delta}(t, s) A^{\sigma}(s)\left(\frac{u}{A}\right)^{\sigma}(s)+H(t, s) \Phi(s) \\
=\left(H_{2}^{\Delta}(t, s) A^{\sigma}(s)+H(t, s) A^{\Delta}(s)\right)\left(\frac{u}{A}\right)^{\sigma}(s) \\
\quad-\gamma H(t, s) A(s) \delta(s)\left[\left(\frac{u}{A}\right)^{\sigma}\right]^{(1+\gamma) / \gamma}(s)
\end{aligned}
$$

Let $\lambda=(1+\gamma) / \gamma$,

$$
\begin{aligned}
a^{\lambda} & =\gamma H(t, s) A(s) \delta(s)\left[\left(\frac{u}{A}\right)^{\sigma}\right]^{(1+\gamma) / \gamma}(s), \\
b^{\lambda-1} & =\frac{\gamma}{1+\gamma} \frac{H_{2}^{\Delta}(t, s) A^{\sigma}(s)+H(t, s) A^{\Delta}(s)}{(\gamma H(t, s) A(s) \delta(s))^{\gamma /(1+\gamma)}} .
\end{aligned}
$$

Using the inequality (a variation of the well-known Young inequality)

$$
\lambda a b^{\lambda-1}-a^{\lambda} \leq(\lambda-1) b^{\lambda}, \quad a, b \geq 0,
$$

we deduce that

$$
\begin{aligned}
& H_{2}^{\Delta}(t, s) A^{\sigma}(s)\left(\frac{u}{A}\right)^{\sigma}(s)+H(t, s) \Phi(s) \\
& \quad \leq \frac{1}{\gamma}\left[\frac{\gamma}{1+\gamma} \frac{H_{2}^{\Delta}(t, s) A^{\sigma}(s)+H(t, s) A^{\Delta}(s)}{(\gamma H(t, s) A(s) \delta(s))^{\gamma /(1+\gamma)}}\right]^{1+\gamma} \\
& \quad=\frac{1}{(1+\gamma)^{1+\gamma}} \frac{\left(H_{2}^{\Delta}(t, s) A^{\sigma}(s)+H(t, s) A^{\Delta}(s)\right)^{1+\gamma}}{(H(t, s) A(s) \delta(s))^{\gamma}} .
\end{aligned}
$$

Therefore, we obtain

$$
\begin{gathered}
\int_{t_{2}}^{t} \frac{H(t, s) A(s) \alpha(s) q(s)}{e_{-d / r}\left(\sigma(s), t_{1}\right)} \Delta s \leq H\left(t, t_{2}\right) u\left(t_{2}\right) \\
+\frac{1}{(1+\gamma)^{1+\gamma}} \\
\quad \cdot \int_{t_{2}}^{t} \frac{\left(H_{2}^{\Delta}(t, s) A^{\sigma}(s)+H(t, s) A^{\Delta}(s)\right)^{1+\gamma}}{(H(t, s) A(s) \delta(s))^{\gamma}} \Delta s,
\end{gathered}
$$

which implies that

$$
\begin{aligned}
& \frac{1}{H\left(t, t_{2}\right)} \int_{t_{2}}^{t}\left[\frac{H(t, s) A(s) \alpha(s) q(s)}{e_{-d / r}\left(\sigma(s), t_{1}\right)}-\frac{1}{(1+\gamma)^{1+\gamma}}\right. \\
& \left.\quad \frac{\left(H_{2}^{\Delta}(t, s) A^{\sigma}(s)+H(t, s) A^{\Delta}(s)\right)^{1+\gamma}}{(H(t, s) A(s) \delta(s))^{\gamma}}\right] \Delta s \\
& \leq u\left(t_{2}\right) .
\end{aligned}
$$

This contradicts (41). Thus, $z^{\Delta}(t)<0$ for $t \in\left[t_{1}, \infty\right)_{\mathbb{T}}$, and so $\lim _{t \rightarrow \infty} z(t)$ exists. By Lemma $3, \lim _{t \rightarrow \infty} x(t)$ exists. The proof is complete.

From Lemma 8 and Theorem 9, we have the following corollary.

Corollary 10. Assume that (37) is satisfied and there exist two functions $A \in C_{r d}^{1}\left(\left[t_{0}, \infty\right)_{\mathbb{T}},(0, \infty)\right)$ and $H \in \mathscr{H}$ such that, for all sufficiently large $t_{1} \in\left[t_{0}, \infty\right)_{\mathbb{T}}$ and for some $t_{2} \in\left[t_{1}, \infty\right)_{\mathbb{T}}$,

$$
\begin{aligned}
& \limsup _{t \rightarrow \infty} \frac{1}{H\left(t, t_{2}\right)} \int_{t_{2}}^{t}\left[\frac{H(t, s) A(s) \alpha(s) q(s)}{e_{-d / r}\left(\sigma(s), t_{1}\right)}\right. \\
& \left.-\frac{1}{(1+\gamma)^{1+\gamma}}\right] \Delta s \\
& \left.\quad \frac{\left(H_{2}^{\Delta}(t, s) A^{\sigma}(s)+H(t, s) A^{\Delta}(s)\right)^{1+\gamma}}{(H(t, s) A(s) \delta(s))^{\gamma}}\right] \Delta \\
& \quad=\infty,
\end{aligned}
$$

where $\alpha$ and $\delta$ are as in Lemma 7. Then (1) is almost oscillatory.

Theorem 11. Assume that (15) holds and there exists a function $A \in C_{r d}^{1}\left(\left[t_{0}, \infty\right)_{\mathbb{T}},(0, \infty)\right)$ such that, for all sufficiently large $t_{1} \in\left[t_{0}, \infty\right)_{\mathbb{T}}$ and for some $t_{2} \in\left[t_{1}, \infty\right)_{\mathbb{T}}$,

$$
\begin{aligned}
\limsup _{t \rightarrow \infty} \int_{t_{2}}^{t}\left[\frac{A(s) \alpha(s) q(s)}{e_{-d / r}\left(\sigma(s), t_{1}\right)}\right. \\
\left.-\frac{1}{(1+\gamma)^{1+\gamma}} \frac{\left(A^{\Delta}(s)\right)^{1+\gamma}}{(A(s) \delta(s))^{\gamma}}\right] \Delta s=\infty,
\end{aligned}
$$

where $\alpha$ and $\delta$ are as in Lemma 7. Then conclusion of Theorem 9 remains intact. 
Proof. Proceeding as in the proof of Theorem 9, assume that $z^{\Delta}(t)>0$ for $t \in\left[t_{1}, \infty\right)_{\mathbb{T}}$. Let $u$ be defined by (25). By virtue of Lemma 7, we arrive at (36). Let $\lambda=(1+\gamma) / \gamma$,

$$
\begin{aligned}
a^{\lambda} & =\gamma A(t) \delta(t)\left[\left(\frac{u}{A}\right)^{\sigma}\right]^{(1+\gamma) / \gamma}(t), \\
b^{\lambda-1} & =\frac{\gamma}{1+\gamma} \frac{A^{\Delta}(t)}{(\gamma A(t) \delta(t))^{\gamma /(1+\gamma)}} .
\end{aligned}
$$

Using (45), we conclude that

$$
u^{\Delta}(t) \leq-\frac{A(t) \alpha(t) q(t)}{e_{-d / r}\left(\sigma(t), t_{1}\right)}+\frac{1}{(1+\gamma)^{1+\gamma}} \frac{\left(A^{\Delta}(t)\right)^{1+\gamma}}{(A(t) \delta(t))^{\gamma}}
$$

which yields

$$
\frac{A(t) \alpha(t) q(t)}{e_{-d / r}\left(\sigma(t), t_{1}\right)}-\frac{1}{(1+\gamma)^{1+\gamma}} \frac{\left(A^{\Delta}(t)\right)^{1+\gamma}}{(A(t) \delta(t))^{\gamma}} \leq-u^{\Delta}(t) .
$$

Let $t_{2} \in\left[t_{1}, \infty\right)_{\mathbb{T}}$. It follows from (53) that

$$
\begin{aligned}
& \int_{t_{2}}^{t}\left[\frac{A(s) \alpha(s) q(s)}{e_{-d / r}\left(\sigma(s), t_{1}\right)}-\frac{1}{(1+\gamma)^{1+\gamma}} \frac{\left(A^{\Delta}(s)\right)^{1+\gamma}}{(A(s) \delta(s))^{\gamma}}\right] \Delta s \\
& \quad \leq u\left(t_{2}\right),
\end{aligned}
$$

which contradicts (50). Therefore, $z^{\Delta}(t)<0$ for $t \in\left[t_{1}, \infty\right)_{\mathbb{T}}$. Along the same lines as in Theorem 9, we complete the proof.

If (37) holds, then we have the following corollary on the basis of Lemma 8 and Theorem 11.

Corollary 12. Assume that (37) is satisfied and there exists a function $A \in C_{r d}^{1}\left(\left[t_{0}, \infty\right)_{\mathbb{T}},(0, \infty)\right)$ such that, for all sufficiently large $t_{1} \in\left[t_{0}, \infty\right)_{\mathbb{T}}$ and for some $t_{2} \in\left[t_{1}, \infty\right)_{\mathbb{T}}$,

$$
\begin{aligned}
& \limsup _{t \rightarrow \infty} \int_{t_{2}}^{t}\left[\frac{A(s) \alpha(s) q(s)}{e_{-d / r}\left(\sigma(s), t_{1}\right)}\right. \\
& \left.-\frac{1}{(1+\gamma)^{1+\gamma}} \frac{\left(A^{\Delta}(s)\right)^{1+\gamma}}{(A(s) \delta(s))^{\gamma}}\right] \Delta s=\infty,
\end{aligned}
$$

where $\alpha$ and $\delta$ are as in Lemma 7. Then conclusion of Corollary 10 remains intact.

Remark 13. If $d \geq 0$, then it is not difficult to see that

$$
0<e_{-d / r}\left(\sigma(t), t_{1}\right) \leq 1
$$

and so (41) and (50) can be simplified to

$$
\begin{gathered}
\limsup _{t \rightarrow \infty} \frac{1}{H\left(t, t_{2}\right)} \int_{t_{2}}^{t}[H(t, s) A(s) \alpha(s) q(s) \\
-\frac{1}{(1+\gamma)^{1+\gamma}} \\
\left.\cdot \frac{\left(H_{2}^{\Delta}(t, s) A^{\sigma}(s)+H(t, s) A^{\Delta}(s)\right)^{1+\gamma}}{(H(t, s) A(s) \delta(s))^{\gamma}}\right] \Delta s \\
=\infty, \quad \sup _{t \rightarrow \infty}^{t}[A(s) \alpha(s) q(s) \\
\left.-\frac{1}{(1+\gamma)^{1+\gamma}} \frac{\left(A^{\Delta}(s)\right)^{1+\gamma}}{(A(s) \delta(s))^{\gamma}}\right] \Delta s=\infty,
\end{gathered}
$$

respectively.

Remark 14. If $h(t) \geq t$, then $\alpha(t)=1$ and we do not impose restrictive condition (15) in our theorems and corollaries.

Remark 15. Our results complement and improve those obtained by Han et al. [14] since we do not impose specific restrictions on $r^{\Delta}$.

\section{Examples}

The following examples are presented to show applications of the main results.

Example 1. Consider the third-order equation

$$
\left(\frac{1}{t} \varphi_{\gamma}\left(z^{\Delta \Delta}(t)\right)\right)^{\Delta}+\frac{\lambda}{t^{2}} \varphi_{\gamma}\left(z^{\Delta \Delta}(t)\right)+\frac{1}{t^{2}} x^{\gamma}\left(\frac{t}{2}\right)=0
$$

where $t \in[1, \infty)_{\mathbb{R}}, z(t)=x(t)-(t-1) x(t-1) /(2 t), \gamma \geq 1$, and $0 \leq \lambda<2$. It is clear that $r(t)=1 / t, p(t)=(t-1) /(2 t)$, $d(t)=\lambda / t^{2}, g(t)=t-1$, and $h(t)=t / 2<t$. Then, $p_{0}=1 / 2$ and $\alpha(t)=1 / 2^{\gamma}$. Let $q(t)=1 / t^{2}$. Since $-d(t) / r(t)=-\lambda / t$, we have

$$
\begin{aligned}
& e_{-d / r}\left(t, t_{0}\right)=\frac{1}{t^{\lambda}} \\
& e_{-d / r}\left(t, t_{1}\right)=\left(\frac{t_{1}}{t}\right)^{\lambda}, \\
& \int_{t_{0}}^{\infty}\left(\frac{e_{-d / r}\left(t, t_{0}\right)}{r(t)}\right)^{1 / \gamma} \Delta t=\int_{1}^{\infty} \frac{1}{t^{(\lambda-1) / \gamma}} d t=\infty,
\end{aligned}
$$

$$
\int_{t_{0}}^{\infty} \frac{q(t) h^{\gamma}(t)}{e_{-d / r}\left(\sigma(t), t_{0}\right)} \Delta t=\frac{1}{2^{\gamma}} \int_{1}^{\infty} t^{\gamma+\lambda-2} d t=\infty,
$$




$$
\begin{aligned}
\delta(t) & =\int_{t_{1}}^{t}\left(\frac{e_{-d / r}\left(s, t_{1}\right)}{r(s)}\right)^{1 / \gamma} \Delta s=t_{1}^{\lambda / \gamma} \int_{t_{1}}^{t} \frac{d s}{s^{(\lambda-1) / \gamma}} \\
& =O\left(t^{(\gamma-\lambda+1) / \gamma}\right) .
\end{aligned}
$$

Hence, assumptions $\left(C_{1}\right)-\left(C_{5}\right)$ and (15) hold. Let $H(t, s)=$ $(t-s)^{2}$ and $A(t)=t^{2}$. If

$$
3 \gamma-4-\lambda+\frac{\lambda-1}{\gamma}<0
$$

we obtain

$$
\begin{aligned}
\limsup _{t \rightarrow \infty} \frac{1}{H\left(t, t_{2}\right)} \int_{t_{2}}^{t}\left[\frac{H(t, s) A(s) \alpha(s) q(s)}{e_{-d / r}\left(\sigma(s), t_{1}\right)}\right. \\
-\frac{1}{(1+\gamma)^{1+\gamma}} \\
\left.\cdot \frac{\left(H_{2}^{\Delta}(t, s) A^{\sigma}(s)+H(t, s) A^{\Delta}(s)\right)^{1+\gamma}}{(H(t, s) A(s) \delta(s))^{\gamma}}\right] \Delta s \\
=\limsup _{t \rightarrow \infty} \frac{1}{\left(t-t_{2}\right)^{2}} \int_{t_{2}}^{t}\left[\frac{(t-s)^{2} s^{\lambda}}{2^{\gamma} t_{1}^{\lambda}}\right. \\
\left.-\frac{O\left(s^{3 \gamma-2+(\lambda-1) / \gamma}\right)}{(1+\gamma)^{1+\gamma}}\right] d s=\infty .
\end{aligned}
$$

That is, (41) is satisfied. By virtue of Theorem 9, we deduce that every solution of (59) either is oscillatory or converges to a finite number asymptotically. Furthermore, if (61) holds and $1 \leq \lambda<2$, then

$$
\int_{t_{0}}^{\infty} \frac{q(t)}{e_{-d / r}\left(\sigma(t), t_{0}\right)} \Delta t=\int_{1}^{\infty} t^{\lambda-2} d t=\infty
$$

which implies that (59) is almost oscillatory by using Corollary 10.

Example 2. Consider the third-order equation

$$
\begin{aligned}
& \left(t \varphi_{\gamma}\left(z^{\Delta \Delta}(t)\right)\right)^{\Delta}-\varphi_{\gamma}\left(z^{\Delta \Delta}(t)\right)+\left(t^{\beta}+t^{\lambda}\right) x^{\gamma}(t+1) \\
& \quad=0
\end{aligned}
$$

where $t \in[2, \infty)_{\mathbb{Z}}, z(t)=x(t)-x(t-2) / t, \beta, \gamma, \lambda \in \mathbb{R}$, and $\gamma \geq 1$. It is easy to see that $r(t)=t, p(t)=-1 / t, d(t)=-1$, $g(t)=t-2$, and $h(t)=t+1>t$. Then, $p_{0}=0$ and $\alpha(t)=1$. Since $-d(t) / r(t)=1 / t$, we get

$$
\begin{aligned}
& e_{-d / r}\left(t, t_{0}\right)=\frac{t}{2}, \\
& e_{-d / r}\left(t, t_{1}\right)=\frac{t}{t_{1}}, \\
& \int_{t_{0}}^{\infty}\left(\frac{e_{-d / r}\left(t, t_{0}\right)}{r(t)}\right)^{1 / \gamma} \Delta t=\frac{1}{2^{1 / \gamma}} \int_{2}^{\infty} \Delta t=\infty, \\
& \delta(t)=\int_{t_{1}}^{t}\left(\frac{e_{-d / r}\left(s, t_{1}\right)}{r(s)}\right)^{1 / \gamma} \Delta s=\frac{1}{t_{1}^{1 / \gamma}} \int_{t_{1}}^{t} \Delta s \\
& =\frac{t-t_{1}}{t_{1}^{1 / \gamma}} .
\end{aligned}
$$

Obviously, conditions $\left(C_{1}\right)-\left(C_{5}\right)$ are satisfied. Let $q(t)=t^{\beta}$ or $t^{\lambda}$ and $A(t)=1$. If $\beta \geq 0$ or $\lambda \geq 0$, then

$$
\begin{gathered}
\int_{t_{0}}^{\infty} \frac{q(t)}{e_{-d / r}\left(\sigma(t), t_{0}\right)} \Delta t \geq \int_{2}^{\infty} \frac{2}{t+1} \Delta t=\infty, \\
\limsup _{t \rightarrow \infty} \int_{t_{2}}^{t}\left[\frac{A(s) \alpha(s) q(s)}{e_{-d / r}\left(\sigma(s), t_{1}\right)}\right. \\
\left.-\frac{1}{(1+\gamma)^{1+\gamma}} \frac{\left(A^{\Delta}(s)\right)^{1+\gamma}}{(A(s) \delta(s))^{\gamma}}\right] \Delta s \geq t_{1} \\
\cdot \limsup _{t \rightarrow \infty} \int_{t_{2}}^{t} \frac{1}{s+1} \Delta s=\infty .
\end{gathered}
$$

That is, both (37) and (55) hold. By Corollary 12, we conclude that (64) is almost oscillatory for $\beta \geq 0$ or $\lambda \geq 0$.

\section{Conflicts of Interest}

The authors declare that they have no conflicts of interest.

\section{Acknowledgments}

This project was supported by the National Natural Science Foundation of China (Grant nos. 11671406, 61503171, and 61403061), China Postdoctoral Science Foundation (Grant no. 2015M582091), Program of Cultivation for Outstanding Young Scholars Sponsored by Guangdong Province (Grant no. ZX03240302), Natural Science Foundation of Shandong Province (Grant nos. ZR2016JL021 and ZR2016AB04), Program of Cultivation for Young Scholars Sponsored by Shunde Polytechnic (Grant no. 2015-KJZX080), Doctoral Scientific Research Foundation of Linyi University (Grant no. LYDX2015BS001), Applied Mathematics Enhancement Program of Linyi University, and Foundation for Young Teachers of Qilu Normal University (Grant no. 2016L0605). 


\section{References}

[1] S. Hilger, Ein Maßkettenkalkül mit Anwendung auf Zentrumsmannigfaltigkeiten [Ph.D. thesis], Universität Würzburg, Würzburg, Germany, 1988.

[2] S. Hilger, "Analysis on measure chains-a unified approach to continuous and discrete calculus," Results in Mathematics. Resultate der Mathematik, vol. 18, no. 1-2, pp. 18-56, 1990.

[3] R. P. Agarwal and M. Bohner, "Basic calculus on time scales and some of its applications," Results in Mathematics, vol. 35, no. 1-2, pp. 3-22, 1999.

[4] R. Agarwal, M. Bohner, D. O’Regan, and A. Peterson, "Dynamic equations on time scales: a survey," Journal of Computational and Applied Mathematics, vol. 141, no. 1-2, pp. 1-26, 2002.

[5] M. Bohner and A. Peterson, Dynamic Equations on Time Scales: An Introduction with Applications, Birkhäuser, Boston, Mass, USA, 2001.

[6] M. Bohner and A. Peterson, Advances in Dynamic Equations on Time Scales, Birkhäuser, Boston, Mass, USA, 2003.

[7] R. P. Agarwal, M. Bohner, T. Li, and C. Zhang, "Hille and Nehari type criteria for third-order delay dynamic equations," Journal of Difference Equations and Applications, vol. 19, no. 10, pp. 15631579, 2013.

[8] R. P. Agarwal, M. Bohner, T. Li, and C. Zhang, "A Philostype theorem for third-order nonlinear retarded dynamic equations," Applied Mathematics and Computation, vol. 249, pp. 527531, 2014.

[9] R. P. Agarwal, M. Bohner, T. Li, and C. Zhang, "Oscillation theorems for fourth-order half-linear delay dynamic equations with damping," Mediterranean Journal of Mathematics, vol. 11, no. 2, pp. 463-475, 2014.

[10] R. P. Agarwal, M. Bohner, S. Tang, T. Li, and C. Zhang, "Oscillation and asymptotic behavior of third-order nonlinear retarded dynamic equations," Applied Mathematics and Computation, vol. 219, no. 8, pp. 3600-3609, 2012.

[11] M. Bohner and T. Li, "Kamenev-type criteria for nonlinear damped dynamic equations," Science China Mathematics, vol. 58, no. 7, pp. 1445-1452, 2015.

[12] T. Candan, "Asymptotic properties of solutions of third-order nonlinear neutral dynamic equations," Advances in Difference Equations, vol. 2014, Article ID 35, 10 pages, 2014.

[13] L. Erbe, T. S. Hassan, and A. Peterson, "Oscillation of third order nonlinear functional dynamic equations on time scales," Differential Equations and Dynamical Systems, vol. 18, no. 1-2, pp. 199-227, 2010.

[14] Z. Han, T. Li, S. Sun, and C. Zhang, "Oscillation behavior of third-order neutral Emden-Fowler delay dynamic equations on time scales," Advances in Difference Equations, Art. ID 586312, 23 pages, 2010.

[15] T. S. Hassan, "Oscillation of third order nonlinear delay dynamic equations on time scales," Mathematical and Computer Modelling, vol. 49, no. 7-8, pp. 1573-1586, 2009.

[16] B. Karpuz, "Nonoscillation and oscillation of second-order linear dynamic equations via the sequence of functions technique," Journal of Fixed Point Theory and Applications, vol. 18, no. 4, pp. 889-903, 2016.

[17] T. Li, R. P. Agarwal, and M. Bohner, "Some oscillation results for second-order neutral dynamic equations," Hacettepe Journal of Mathematics and Statistics, vol. 41, no. 5, pp. 715-721, 2012.

[18] T. Li, Z. Han, S. Sun, and Y. Zhao, "Oscillation results for third order nonlinear delay dynamic equations on time scales,"
Bulletin of the Malaysian Mathematical Sciences Society. Second Series, vol. 34, no. 3, pp. 639-648, 2011.

[19] Y.-C. Qiu, "Oscillation criteria of third-order nonlinear dynamic equations with nonpositive neutral coefficients on time scales," Advances in Difference Equations, vol. 2015, Article ID 299, 16 pages, 2015.

[20] Y.-C. Qiu and Q.-R. Wang, "New oscillation results of secondorder damped dynamic equations with $p$-Laplacian on time scales," Discrete Dynamics in Nature and Society. An International Multidisciplinary Research and Review Journal, vol. 2015, Article ID 709242, 9 pages, 2015.

[21] S. H. Saker, R. P. Agarwal, and D. O’Regan, "Oscillation of second-order damped dynamic equations on time scales," Journal of Mathematical Analysis and Applications, vol. 330, no. 2, pp. 1317-1337, 2007.

[22] M. T. Şenel and N. Utku, "Oscillation criteria for third-order neutral dynamic equations with continuously distributed delay," Advances in Difference Equations, vol. 2014, Article ID 220, 15 pages, 2014.

[23] Z.-H. Yu and Q.-R. Wang, "Asymptotic behavior of solutions of third-order nonlinear dynamic equations on time scales," Journal of Computational and Applied Mathematics, vol. 225, no. 2, pp. 531-540, 2009. 


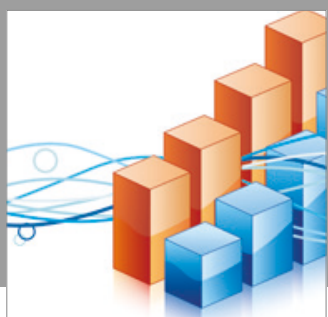

Advances in

Operations Research

vatersals

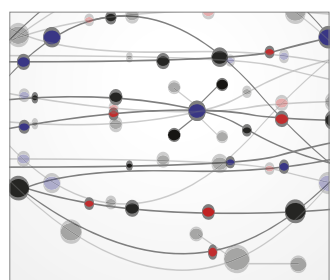

\section{The Scientific} World Journal
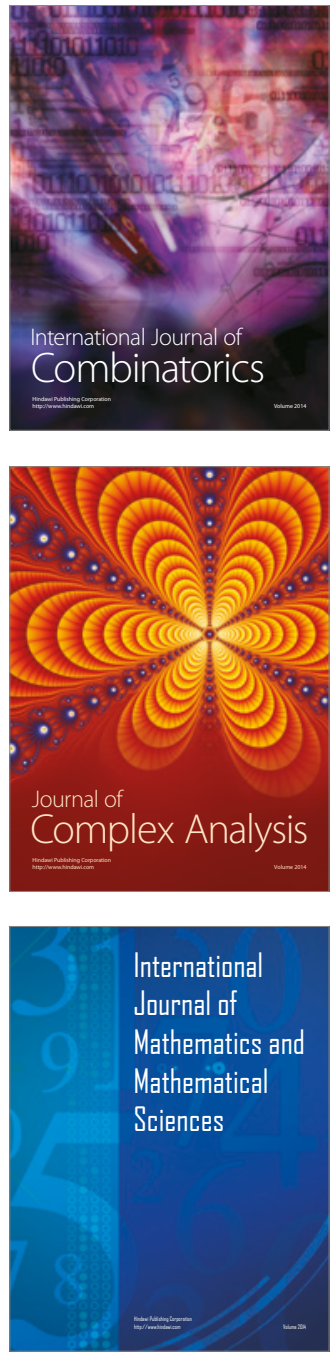
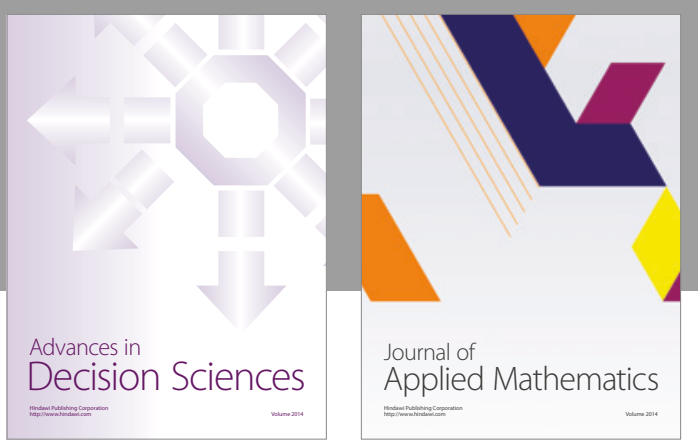

Algebra

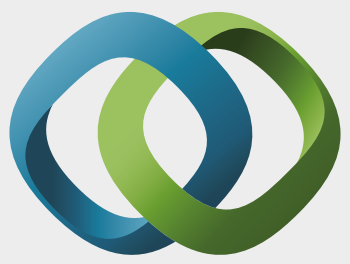

\section{Hindawi}

Submit your manuscripts at

https://www.hindawi.com
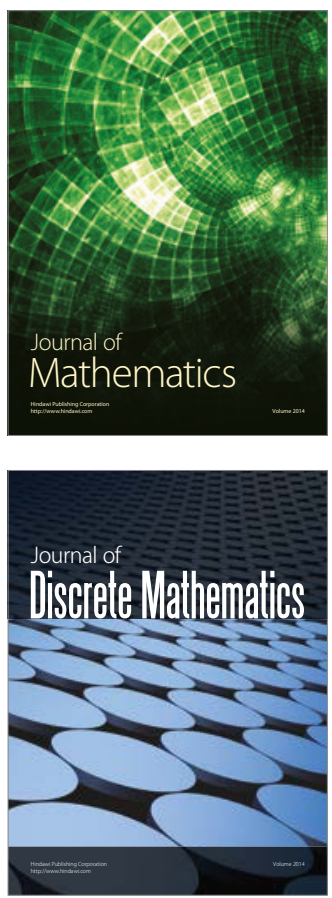

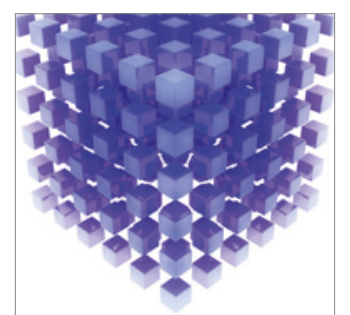

Mathematical Problems in Engineering
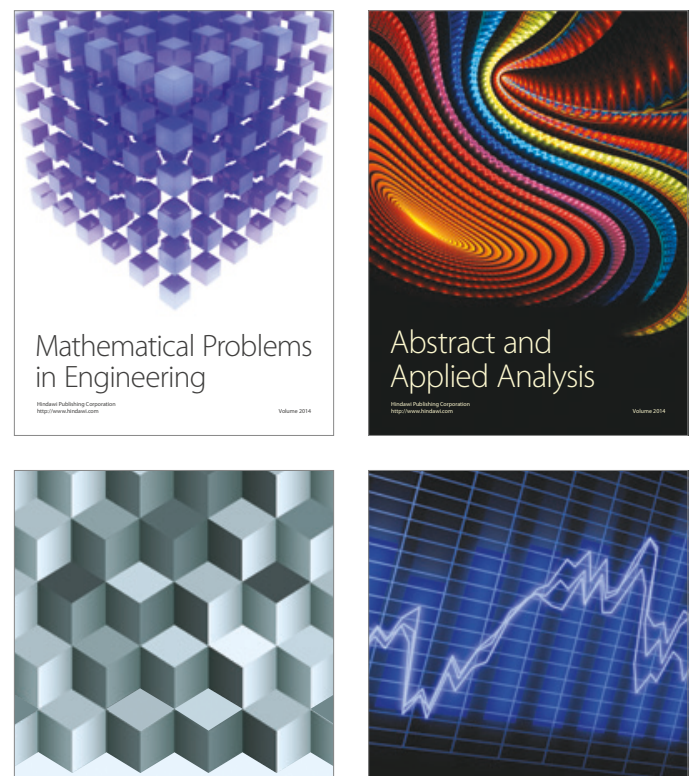

Journal of

Function Spaces

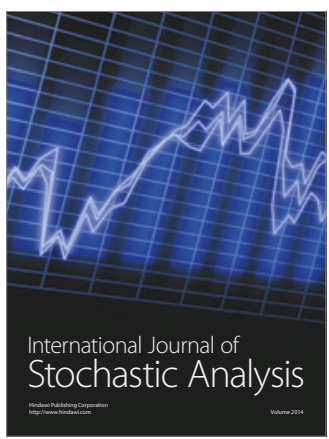

Probability and Statistics
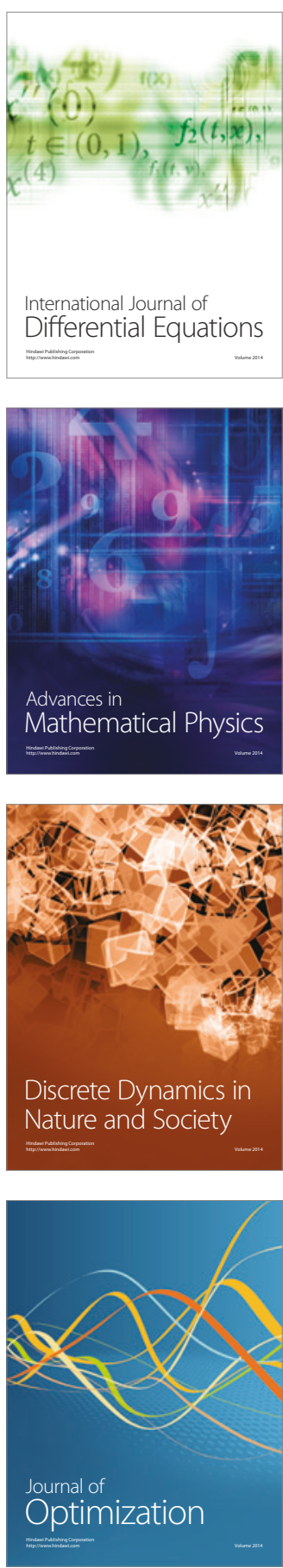